University of New Hampshire

University of New Hampshire Scholars' Repository

Physics Scholarship

Physics

$4-2014$

\title{
Quantifying the radiation belt seed population in the 17 March 2013 electron acceleration event
}

\author{
A. J. Boyd \\ University of New Hampshire - Main Campus \\ Harlan E. Spence \\ University of New Hampshire, harlan.spence@unh.edu \\ S. Claudepierre \\ Aerospace Corporation \\ Joseph F. Fennell \\ Aerospace Corporation \\ J. B. Blake \\ Aerospace Corporation
}

See next page for additional authors

Follow this and additional works at: https://scholars.unh.edu/physics_facpub

Part of the Physics Commons

\section{Recommended Citation}

Boyd, A. J., H. E. Spence, S. G. Claudepierre, J. F. Fennell, J. B. Blake, D. N. Baker, G. D. Reeves, and D. L.Turner (2014), Quantifying the radiation belt seed population in the March 17, 2013 electron acceleration event, Geophys. Res. Lett., 41, 2275-2281, doi:10.1002/2014GL059626.

This Article is brought to you for free and open access by the Physics at University of New Hampshire Scholars' Repository. It has been accepted for inclusion in Physics Scholarship by an authorized administrator of University of New Hampshire Scholars' Repository. For more information, please contact Scholarly.Communication@unh.edu. 


\section{Authors}

A. J. Boyd, Harlan E. Spence, S. Claudepierre, Joseph F. Fennell, J. B. Blake, D. N. Baker, Geoffrey Reeves, and D. L. Turner 


\section{Geophysical Research Letters}

\author{
RESEARCH LETTER \\ 10.1002/2014GL059626 \\ Key Points: \\ - Quantification of phase space density \\ gradients inside geostationary orbit \\ - Clear differences between \\ the source of low energy and \\ relativistic electrons \\ - Clear observations of how the \\ acceleration process evolves \\ in energy
}

\section{Supporting Information: \\ - Readme \\ - Figure S1}

Correspondence to:

A. J. Boyd,

a.j.boyd@unh.edu

\section{Citation:}

Boyd, A. J., H. E. Spence, S. G. Claudepierre, J. F. Fennell, J. B. Blake, D. N. Baker, G. D. Reeves, and D. L. Turner (2014), Quantifying the radiation belt seed population in the March 17, 2013 electron acceleration event, Geophys. Res. Lett., 41, 2275-2281, doi:10.1002/2014GL059626.

\section{Received 13 FEB 2014} Accepted 1 APR 2014 Accepted article online 2 APR 2014 Published online 15 APR 2014

\section{Quantifying the radiation belt seed population in the 17 March 2013 electron acceleration event}

\author{
A. J. Boyd ${ }^{1}$, H. E. Spence ${ }^{1}$, S. G. Claudepierre², J. F. Fennell ${ }^{2}$, J. B. Blake², D. N. Baker ${ }^{3}$, \\ G. D. Reeves ${ }^{4}$, and D. L. Turner ${ }^{5}$
}

${ }^{1}$ Institute for the Study of Earth, Oceans, and Space, University of New Hampshire, Durham, New Hampshire, USA, ${ }^{2}$ Space Sciences Department, The Aerospace Corporation, Los Angeles, California, USA, ${ }^{3}$ Laboratory for Atmospheric and Space Physics, University of Colorado Boulder, Boulder, Colorado, USA, ${ }^{4}$ Space Science and Applications, Los Alamos National Laboratory, Los Alamos, New Mexico, USA, ${ }^{5}$ Department of Earth and Space Sciences, University of California, Los Angeles, California, USA

\begin{abstract}
We present phase space density (PSD) observations using data from the Magnetic Electron lon Spectrometer instrument on the Van Allen Probes for the 17 March 2013 electron acceleration event. We confirm previous results and quantify how PSD gradients depend on the first adiabatic invariant. We find a systematic difference between the lower-energy electrons ( $<\mathrm{few} 100 \mathrm{keV}$ ) originating from the plasma sheet and higher-energy electrons $>1 \mathrm{MeV}$ with a source region within the radiation belts. Our observations show that the source process begins with enhancements to the $10 \mathrm{~s}-100 \mathrm{~s}$ keV energy seed population, followed by enhancements to the $>1 \mathrm{MeV}$ population and eventually leading to enhancements in the multi-MeV electron population. These observations provide the clearest evidence to date of the timing and nature of the radial transport of a $100 \mathrm{~s}$ keV electron seed population into the heart of the outer belt and subsequent local acceleration of those electrons to higher radiation belt energies.
\end{abstract}

\section{Introduction}

One of the primary goals of the recently launched Van Allen Probes mission is to understand the acceleration process for relativistic electrons in the radiation belts. Based on theory, the two main processes for this acceleration are radial diffusion and local acceleration via wave-particle interactions [Reeves et al., 2013]. Local acceleration has been shown to be capable of quickly producing enhancements in relativistic electrons. Reeves et al. [2013] recently provided evidence of local acceleration of MeV electrons for an event on 8-9 October 2012, but that paper did not examine, in any detail, the relative effects of local acceleration and radial diffusion as a function of energy. This leaves open the question of the origin and evolution of the initial, lower-energy electrons (10s to $100 \mathrm{~s} \mathrm{keV)} \mathrm{that} \mathrm{serve} \mathrm{as} \mathrm{a} \mathrm{"seed"} \mathrm{population} \mathrm{for} \mathrm{the} \mathrm{radiation} \mathrm{belts} \mathrm{[Baker}$ et al., 1998; Baker and Kanekal, 2008].

To examine these acceleration processes, phase space density (PSD) in adiabatic coordinates $\left(\mu, K, L^{*}\right)$ is needed to clearly separate adiabatic and nonadiabatic effects. For a fixed first invariant $\mu$ and second invariant $K$, the radial gradients of PSD as a function of $L^{*}$ and time reveal key aspects of the acceleration, transport, and loss of electron populations. Examining the radial gradients in phase space density at different energies (or different $\mu$ ) can differentiate the effects of radial transport of seed electrons from the magnetotail from the effects of local acceleration of those electrons to higher energies. Many studies, beginning with Selesnick et al. [1997] have shown that there are $\mu$-dependent radial gradients for radiation belt electrons. Recently, Chen et al. [2005], Turner and Li [2008], Kim et al. [2010], and Turner et al. [2012] have examined the PSD gradients beyond geostationary orbit and found that $\sim 200 \mathrm{MeV} / \mathrm{G}$ is the typical transition point between positive and negative gradients. Examining these PSD gradients with the instrumentation onboard the Van Allen Probes and seeing how they evolve in time enables us to identify the seed population and explore the role it plays in the local acceleration process.

In the following sections, we introduce the data sets that were used and discuss the method we employed to calculate PSD and estimate the error in this calculation. Then we show the initial results of this study looking at both the radial PSD gradients as a function of $\mu$ and how the PSD distribution evolves as a function of time. 


\section{Data}

The twin Van Allen Probes spacecraft were launched on 30 August 2012 with a comprehensive instrument suite to examine the particles, fields, and waves in the radiation belts [Mauk et al., 2012]. The probes were launched in low inclination, highly elliptical orbits with apogees near $5.8 R_{E}$. For this study, we use energetic electron fluxes from the Magnetic Electron Ion Spectrometer (MagEIS) [Blake et al., 2013], part of the Energetic Particle Composition and Thermal Plasma Suite (ECT) [Spence et al., 2013], and magnetic field measurements from the Energetic and Magnetic Field Instrument Suite and Integrated Science (EMFISIS) [Kletzing et al., 2013] magnetometer.

The MagEIS instrument provides pitch angle resolved particle fluxes for electrons with energies of 20-4000 keV. The MagEIS units on both probes ( $A$ and B) contain identical instrumentation and from here on will be referred to as MagEIS-A and MagEIS-B, respectively for each spacecraft. The instrument is divided into four detectors, a low-energy unit (20-240 keV), two medium-energy units to provide additional pitch angle coverage (M35 and M75; 80-1200 keV), and a high-energy unit (800-4800 keV). For this study, to avoid complications with merging the two medium-energy data sets, we use data from low-energy, M75, and high-energy units, which provide identical pitch angle coverage.

For this study, we used MagEIS data to calculate the phase space density for a set of $\mu$ and $K$ values around 17 March 2013. This period includes a strong acceleration event on 17 and 18 March following a coronal mass ejection (CME) impact on 17 March. For a detailed look at the multi-MeV electron response to this event, see the companion paper by Baker et al. [2014]. The solar wind conditions for this event are shown in Figures $1 \mathrm{a}-1 \mathrm{~d}$. This was a moderately strong storm with a minimum Dst index of $-130 \mathrm{nT}$. Beginning around 8:00 UT on 17 March, there was a period of $18 \mathrm{~h}$ of continuous southward interplanetary magnetic field (IMF) $B z$. During this time there was considerable substorm activity, indicated by the elevated Auroral Electrojet $(A E)$ index. The spacecraft apogees for this event were near midnight magnetic local time, and both spacecraft were in nearly identical orbits, with Probe-A trailing Probe-B by about $1 \mathrm{~h}$. This configuration provided an opportunity to get observations of key spatial regions with excellent temporal resolution.

\section{Methodology}

To calculate the phase space density we follow the basic method outlined in Chen et al. [2005] and Turner and $L i$ [2008]. Given the differential particle flux $j(E, \alpha, t)$, we can convert to $\operatorname{PSD} f$, using the following fully relativistic formula:

$$
f=3.325 \times 10^{-8} \frac{j(E)}{E\left(E+2 m_{0} c^{2}\right)}\left[\left(\frac{\mathrm{c}}{\mathrm{MeV} \mathrm{cm}}\right)^{3}\right]
$$

where $j$ is the particle flux in \#/cm² sr s keV, $E$ is the particle's kinetic energy in $\mathrm{MeV}, m_{0} c^{2}$ is the electron rest mass in $\mathrm{MeV}$, and the numerical factor converts to the units shown in brackets.

The next step is to express the PSD in adiabatic coordinates $\left(\mu, K, L^{*}\right)$. The second and third invariants $K$ and $L^{*}$ are provided for 18 different pitch angles by the ECT Science Operations Center. For this study, we use the magnetic ephemeris calculated using the TS04D magnetic field model [Tsyganenko and Sitnov, 2005]. Choosing a fixed $K$ value, we interpolate $K(\alpha)$ to get the appropriate pitch angle for a given $K$ value for each time step. Then, for each energy channel, we interpolate $f(\alpha)$ to determine the phase space density for that $K$ value. We then use a univariate spline interpolation on $f(E)$ and select the correct energy using the following definition of $\mu$ :

$$
\mu=\frac{E\left(E+2 m_{0} c^{2}\right) \sin ^{2}(\alpha)}{2 B m_{0} c^{2}}\left[\frac{\mathrm{MeV}}{\mathrm{G}}\right]
$$

where $B$ is the magnetic field from the EMFISIS magnetometer. Finally, we transform from spatial coordinates (e.g., satellite location) to $L^{*}$ (as a function of $K$ ) to obtain PSD in magnetic coordinates, $f\left(\mu, K, L^{*}\right)$.

To estimate the error in PSD, we use phase space density matching [Chen et al., 2005]. This process involves comparing the PSD between MagEIS-A and MagEIS-B when they are simultaneously measuring electrons at the same $\mu, K$, and $L^{*}$. This process and the results from the Relativistic Electron Proton Telescope (REPT) [Baker et al., 2012] instrument are discussed in Morley et al. [2013]. For this study we use the period from 19 to 21 March to estimate the errors in PSD. For this period, conjunctions in $L^{*}$ occurred close to apogee. We 

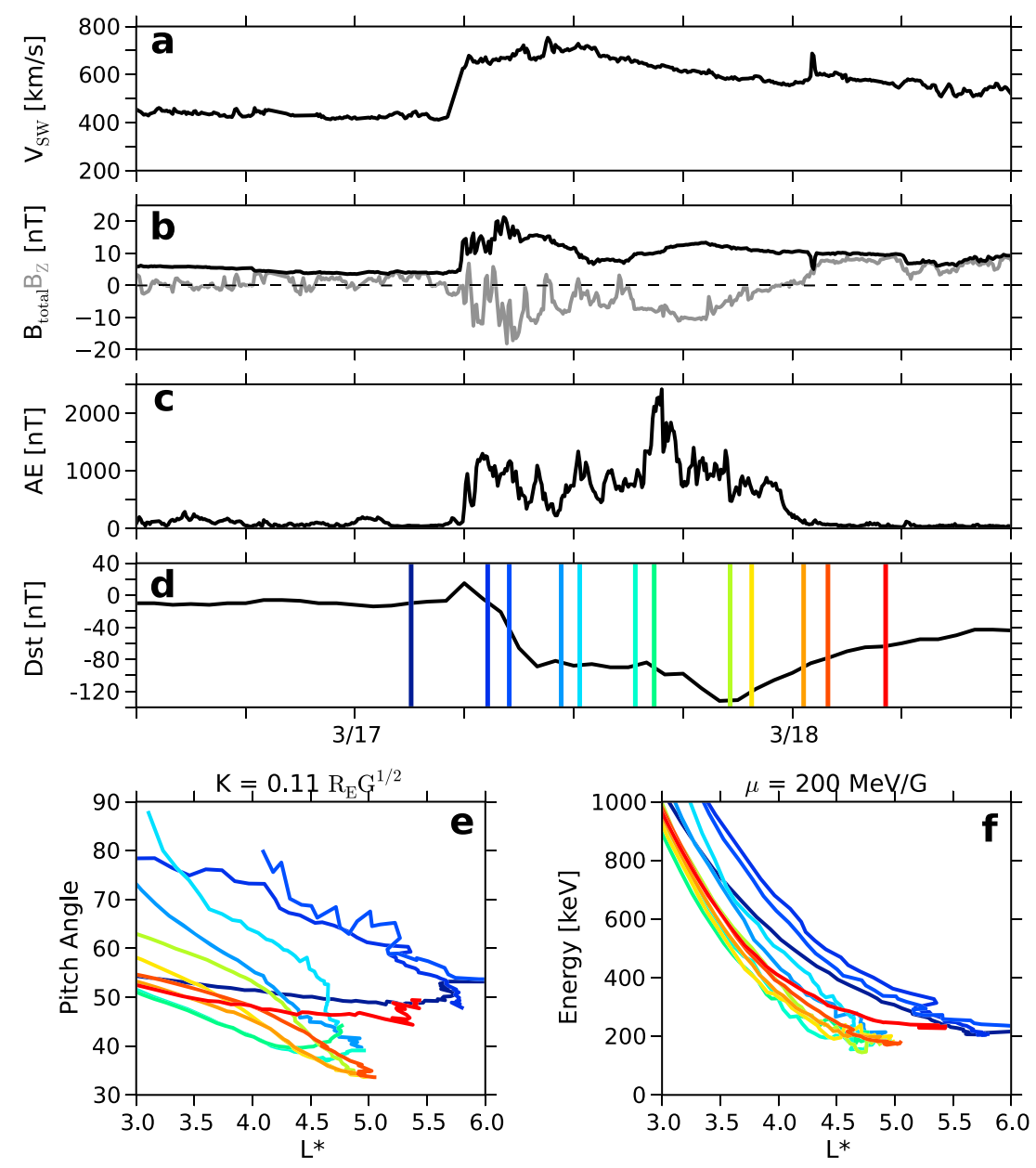

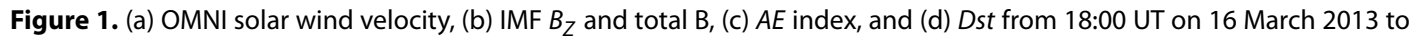
12:00 UT on 18 March 2013. (e) Pitch angle for $K=0.11 R_{E} G^{1 / 2}$ and (f) electron energy for first invariant $\mu=200 \mathrm{MeV} / \mathrm{G}$ as a function of $L^{*}$. The colors of the vertical lines on the Dst plot correspond to the colors of the passes plotted in Figures $1 \mathrm{e}$ and $1 \mathrm{f}$ as well as Figure 3.

found that these errors were somewhat $\mu$ dependent but were consistent with the REPT PSD errors found by Morley et al. [2013], with an average error factor of 1.3 and larger errors at the highest $\mu$ values.

\section{Results}

Figure 2 shows the MagEIS-B PSD for a range of $\mu$ values for a single inbound pass. The $\mu$-dependent error estimates at $10 \mathrm{~min}$ intervals are shown as error bars on each curve. This pass covered a period from 23:10 UT on 17 March to 3:02 UT on 18 March, placing it early in the recovery phase of the storm. Plotting this pass as a function of $L^{*}$ provides a snapshot of the outer radiation belt to observe the PSD gradients as a function of $\mu$. We chose to look at the gradients during this pass to characterize the structure of the belt immediately after the acceleration event.

From Figure 2 it is clear that the PSD has a positive gradient in $L^{*}$ for $\mu<200 \mathrm{MeV} / \mathrm{G}$ and flat or negative gradients for $\mu>200 \mathrm{MeV} / \mathrm{G}$. This transition at $200 \mathrm{MeV} / \mathrm{G}$ is consistent with previous estimates by Turner and $L i$ [2008], Kim et al. [2010], and Turner et al. [2012]. While this study does confirm previous results, these results also represent the first time these gradients have been quantified for a broad range of $\mu$ in the heart of the radiation belts during storm time. Previous studies have been limited to measurements that were either beyond GEO, off-equatorial, or at higher $\mu$. This $\mu$ dependence in the gradients persists through most of the recovery phase, until outward radial diffusion moves the PSD peaks beyond the Van Allen Probe apogee, resulting in positive gradients for all $\mu$ values. During quiet times the PSD peaks for relativistic electrons should typically be at or beyond the Van Allen Probe apogee [Turner et al., 2012], which makes it difficult 


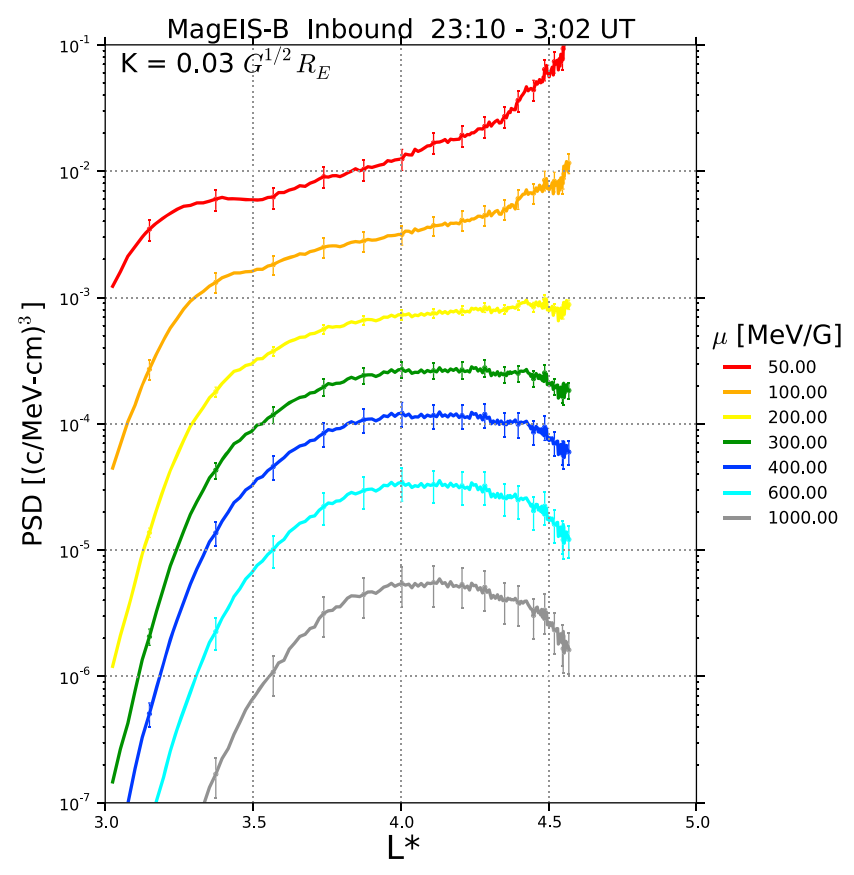

Figure 2. Radial PSD profiles for various $\mu$ values during one inbound pass observed by MagEIS-B. Error bars are shown at 10 min intervals. This pass corresponds to the orange vertical line in the Dst plot in Figure 1. to locate the transition point between positive and negative gradients. However, the same transition at $\sim 200 \mathrm{MeV} / \mathrm{G}$ is also seen during the recovery phase of the $8-9$ October 2012 storm.

As discussed in Green and Kivelson [2004], PSD profiles tend to be peaked in their source regions. Therefore, since the $<200 \mathrm{MeV} / \mathrm{G}$ population has a positive PSD gradient, this suggests that the source region is beyond the Van Allen Probe apogee and these particles likely come from the plasma sheet. Conversely, the PSD profile of larger $\mu$ particles is peaked within (and therefore has a source within) the radiation belt. As suggested by Turner et al. [2012], the transition of these gradients around $200 \mathrm{MeV} / \mathrm{G}$ is consistent with the theory of local acceleration by wave-particle interaction with chorus waves. The $<200 \mathrm{MeV} / \mathrm{G}$ particles that originate in the plasma sheet can be locally

accelerated by interactions with waves to produce the higher $\mu$ particles that have a source within the radiation belt. A value of $\mu=200 \mathrm{MeV} / \mathrm{G}$ corresponds to an energy of $400 \mathrm{keV}$ at $L^{*}=4$ (see Figure 1f). Horne et al. [2005] showed that this is a critical energy for acceleration by chorus waves, where energies below it are scattered into the loss cone faster than they are accelerated. This suggests that this $\mu<200 \mathrm{MeV} / \mathrm{G}$ population could be the "seed" population for the relativistic electrons in the outer radiation belt.

Next, we explore the evolution of the PSD over the course of the 17/18 March storm. In Figure 3, we plot the PSD from each inbound and outbound pass for six values of $\mu$ between 03:05 UT on 17 March, just before the storm, and 6:17 UT on 18 March, during the recovery phase. This allows us to examine how the PSD profile evolves in time over the course of the storm. All the given times correspond to the $L^{*}=4.2$ crossing time and are marked and color coded on the Dst plot in Figure 1 for context. For this section we looked at a higher $K$ value $\left(0.11 R_{E} G^{1 / 2}\right)$ in order to have sufficient coverage in $L^{*}$ for the whole time period.

It is clear that, over the course of the storm, the PSD evolves very differently for different $\mu$ values. For $\mu=50 \mathrm{MeV} / \mathrm{G}$ and $\mu=100 \mathrm{MeV} / \mathrm{G}$, there is a large, sudden increase in PSD near the Van Allen Probes apogee, i.e., $L^{*}>5.5$ for the 3:05 UT orbit (outbound). This feature is likely associated with a substorm injection seen by GOES around this time (see the supporting information) and identified by the peak in $A E$ at 8:00 UT. In the two subsequent inbound orbits, the peak emerges and grows inside $L^{*}=5.5$ first for Probe $A$, the trailing spacecraft. By the time the next two outbound passes come $3.5 \mathrm{~h}$ later, the PSD has been enhanced at all $L^{*}$. For the $\mu=100 \mathrm{MeV} / \mathrm{G}$ population, this is followed by enhancement over a wide range of $L^{*}$, indicative of acceleration by radial diffusion. Later in the event, beginning at 15:00 UT, there is another enhancement seen at $L^{*}<3.2$ for $\mu=50 \mathrm{MeV} / \mathrm{G}$. This is associated with another substorm injection, coinciding with the peak in $A E$ and observed by GOES around the same time. This reinforces the notion that injections of fresh lower-energy electrons from the inner magnetotail are an important initial step that precedes local acceleration to higher energies.

The direct effects of the injections were not seen for $\mu>300 \mathrm{MeV} / \mathrm{G}$. Instead, there are clear PSD peaks that form at low $L^{*}$ and then increase with time. During the 11:29/12:20 UT passes, the $50 \mathrm{MeV} / \mathrm{G}$ population has already been enhanced and the $100 \mathrm{MeV} / \mathrm{G}$ begins to show signs of enhancement, and the $>500 \mathrm{MeV} / \mathrm{G}$ population shows signs of a strong loss process, which was related to a flux dropout during the main phase of this storm. On the following orbit 15:23/16:25 UT, the $100 \mathrm{MeV} / \mathrm{G}$ population continues to enhance, and 


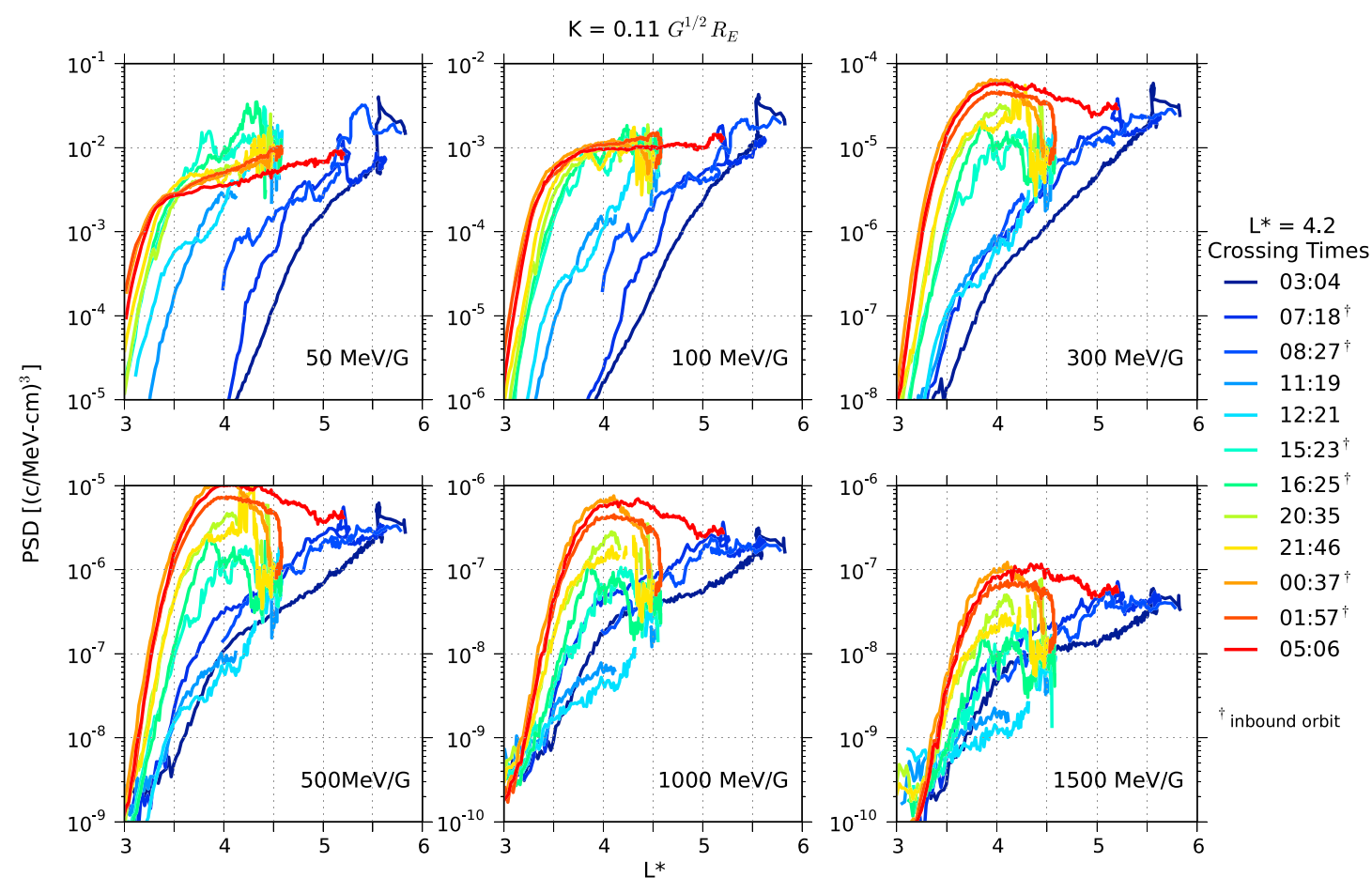

Figure 3. PSD distributions for each inbound and outbound pass between 3:05 UT on 17 March and 5:07 UT on 18 March. All time labels are the $L^{*}=4.2$ crossing time. Inbound passes are denoted by dagger. The color of each of the passes correspond to the vertical lines in the Dst plot in Figure 1.

300-1000 MeV/G population begins to show peaks in PSD at low $L^{*}$. Finally, on 20:35/21:46 UT orbit, the $1500 \mathrm{MeV} / \mathrm{G}$ population begins to show a significant PSD peak. Although not shown here, the REPT PSD at higher $\mu$ is qualitatively similar to the PSD at $\mu=1500 \mathrm{MeV} / \mathrm{G}$.

As shown in Green and Kivelson [2004] and Reeves et al. [2013], an increasing phase space density peak may be an indication of local acceleration. Another possibility for the increasing PSD peaks is an on-off source at high $L^{*}$. However, for this storm such a source is unlikely. While the acceleration process is active, there were two periods when neither of the spacecraft were beyond the observed PSD peak $\left(L^{*}>4.4\right)$. During the first, 15:53-20:41 UT, substorm injections were observed by GOES (see the supporting information). However, for the second, 1:42-5:15 UT, there was no evidence of substorm injections in the GOES observations making an on-off source unlikely. Additionally, none of the injections seen earlier in the storm caused increases in the $>300 \mathrm{MeV} / \mathrm{G}$ electrons. It is unlikely that this on-off source for $>300 \mathrm{MeV} / \mathrm{G}$ electrons would only operate during the time periods when one of the spacecraft was not in a position to observe it. Another possibility is that errors in the PSD calculation could account for some of the increases. We refer the reader to Green and Kivelson [2004] and Reeves et al. [2013, supporting information] for details on how errors in the global magnetic field models can lead to errors in PSD. It is unlikely that these errors can account for the nearly 2 orders of magnitude increase in PSD seen over the course of the storm. While it is not possible to completely eliminate these other possibilities, local acceleration is the most likely explanation for the growing PSD peaks observed during this storm.

This set of observations shows clear evidence of a sequence of acceleration occurring sequentially with the injection of a lower-energy seed population from the plasma sheet radially inward. That process accelerates electrons up to a few hundred keV in the heart of the radiation belts [Baker et al., 1998; Baker and Kanekal, 2008]. The higher-energy electrons later show increasing PSD peaks, showing evidence of local acceleration. These two different acceleration processes for the populations below and above $\mu \sim 200 \mathrm{MeV} / \mathrm{G}$ are clearly shown in Figure 3 and are consistent with a $<200 \mathrm{MeV} / \mathrm{G}$ seed population preceding subsequent local acceleration to enhance the relativistic populations within the heart of the outer belt.

This timing of this acceleration process is shown more clearly in Figure 4, which shows the ratio of PSD to the prestorm level (3:05 UT orbit) at $L^{*}=4$. This figure clearly shows that at $L^{*}=4$, the initial acceleration of 


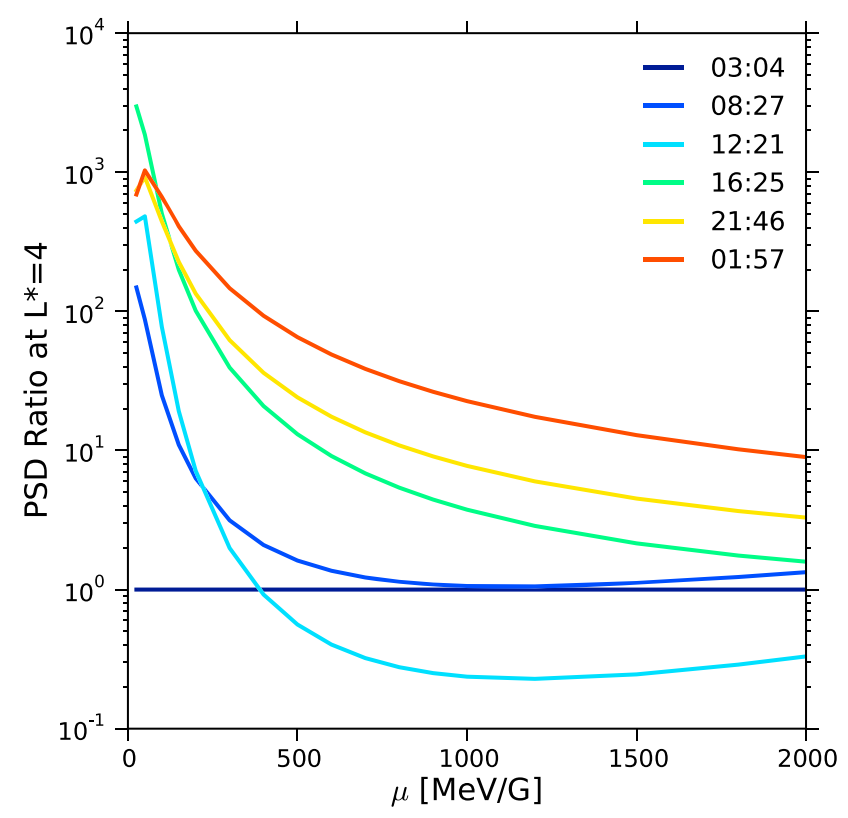

Figure 4. The ratio of the PSD at $L^{*}=4$ to the prestorm level observed during the 3:05 UT orbit. For simplicity, only the PSD from MagEIS-A is shown here. The colors of the curves correspond to those in Figures 1 and 3. the seed electrons is followed by a strong loss of the $>500 \mathrm{MeV} / \mathrm{G}$ population. This is then followed by acceleration at all $\mu$ values. The acceleration process for the relativistic electrons does not seem to begin until this seed population is in place at low $L^{*}$ values. After the seed population is in place, the acceleration up to multi-MeV occurs over a period of less than $12 \mathrm{~h}$. More specific timescales for this acceleration process is difficult to determine without greater time resolution. However, the orbital configuration and instrumentation from the Van Allen Probes for this event allowed the observation of this progression from seed electrons up to multi-MeV energies with high precision and with excellent and unprecedented resolution and specification of the adiabatic coordinates.

\section{Conclusions}

We present electron PSD distributions from MagEIS over a broad range of $\mu$ values for the 17 March 2013 electron acceleration event. We have quantified the $\mu$ dependence of PSD gradients inside GEO for the first time, confirming the results of previous studies, with $\mu<200 \mathrm{MeV} / \mathrm{G}$ showing positive gradients and $\mu>200 \mathrm{MeV} / \mathrm{G}$ showing peaks in the heart of the radiation belts and flat or negative gradients at higher $L^{*}$. We combine these observations with results showing local acceleration in the heart of the radiation belts [e.g., Reeves et al., 2013]. Taking advantage of the comprehensive measurements available from the Van Allen Probes, we can now connect the two steps of the acceleration process with a single, unified, set of observations. Our results demonstrate that the acceleration process for this storm begins, first, with the injection of the seed population from the plasma sheet and is followed by local acceleration up to multi-MeV energies. Future work will explore a range of conditions in different events to better understand the role of the seed population in determining which storms lead to radiation belt enhancement events and which do not.

\section{Acknowledgments}

This work was supported by RBSP-ECT funding provided by JHU/APL contract 967399 under NASA's Prime contract NAS5-01072. D.L.T. is thankful for funding from NASA (THEMIS contract NAS5-02099 and grant NNX12AJ55G). The OMNI data were obtained from the GSFC/SPDF OMNIWeb interface at http://omniweb.gsfc.nasa.gov. The GOES data were provided by NGDC and SWPC at NOAA and obtained from the GSFC/SPDF OMNIWeb interface. A.J.B. would like to thank the developers of the SpacePy tools (http:// spacepy.lanl.gov).

The Editor thanks Andrew Yau and two anonymous reviewers for their assistance in evaluating this paper.

\section{References}

Baker, D., and S. Kanekal (2008), Solar cycle changes, geomagnetic variations, and energetic particle properties in the inner magnetosphere, J. Atmos. Sol. Terr. Phys., 70(2-4), 195-206, doi:10.1016/j.jastp.2007.08.031.

Baker, D., X. Li, J. Blake, and S. Kanekal (1998), Strong electron acceleration in the Earth's magnetosphere, Adv. Space Res., 21(4), 609-613, doi:10.1016/S0273-1177(97)00970-8.

Baker, D., et al. (2012), The relativistic electron-proton telescope (REPT) instrument on board the radiation belt storm probes (RBSP) spacecraft: Characterization of Earth's radiation belt high-energy particle populations, Space Sci. Rev., 179, 337-381, doi:10.1007/s11214-012-9950-9.

Baker, D., et al. (2014), Gradual diffusion and punctuated phase space density enhancements of highly relativistic electrons: Van Allen Probes observations, Geophys. Res. Lett., 41, 1351-1358, doi:10.1002/2013GL058942.

Blake, J. B., et al. (2013), The Magnetic Electron Ion Spectrometer (MagEIS) instruments aboard the Radiation Belt Storm Probes (RBSP) spacecraft, Space Sci. Rev., 179, 383-421, doi:10.1007/s11214-013-9991-8.

Chen, Y., R. H. W. Friedel, G. D. Reeves, T. G. Onsager, and M. F. Thomsen (2005), Multisatellite determination of the relativistic electron phase space density at geosynchronous orbit: Methodology and results during geomagnetically quiet times, J. Geophys. Res., 110, A10210, doi:10.1029/2004JA010895.

Green, J. C., and M. G. Kivelson (2004), Relativistic electrons in the outer radiation belt: Differentiating between acceleration mechanisms, J. Geophys. Res., 109, A03213, doi:10.1029/2003JA010153.

Horne, R. B., R. M. Thorne, S. A. Glauert, J. M. Albert, N. P. Meredith, and R. R. Anderson (2005), Timescale for radiation belt electron acceleration by whistler mode chorus waves, J. Geophys. Res., 110, A03225, doi:10.1029/2004JA010811. 
Kim, H.-J., E. Zesta, K.-C. Kim, Y. Shprits, Y. Shi, and L. R. Lyons (2010), Estimation of radial gradients of phase space density from POLAR observations during a quiet period prior to a sudden solar wind dynamic pressure enhancement, J. Geophys. Res., 115, A12249, doi:10.1029/2010JA015722.

Kletzing, C. A., et al. (2013), The Electric and Magnetic Field Instrument Suite and Integrated Science (EMFISIS) on RBSP, Space Sci. Rev., 179, 127-181, doi:10.1007/s11214-013-9993-6.

Mauk, B. H., N. J. Fox, S. G. Kanekal, R. L. Kessel, D. G. Sibeck, and A. Ukhorskiy (2012), Science objectives and rationale for the radiation belt storm probes mission, Space Sci. Rev., 179, 3-27, doi:10.1007/s11214-012-9908-y.

Morley, S. K., M. G. Henderson, G. D. Reeves, R. H. W. Friedel, and D. N. Baker (2013), Phase Space Density matching of relativistic electrons using the Van Allen Probes: REPT results, Geophys. Res. Lett., 40, 4798-4802, doi:10.1002/grl.50909.

Reeves, G. D., et al. (2013), Electron acceleration in the heart of the Van Allen radiation belts., Science, 341(6149), 991-994, doi:10.1126/science.1237743.

Selesnick, R. S., J. B. Blake, W. A. Kolasinski, and T. A. Fritz (1997), A quiescent state of 3 to 8 MeV radiation belt electrons, Geophys. Res. Lett., 24(11), 1343-1346, doi:10.1029/97GL51407.

Spence, H. E., et al. (2013), Science goals and overview of the Energetic Particle, Composition, and Thermal Plasma (ECT) suite on NASAs Radiation Belt Storm Probes (RBSP) mission, Space Sci. Rev., 179, 311-336, doi:10.1007/s11214-013-0007-5.

Tsyganenko, N. A., and M. I. Sitnov (2005), Modeling the dynamics of the inner magnetosphere during strong geomagnetic storms, J. Geophys. Res., 110, A03208, doi:10.1029/2004JA010798.

Turner, D. L., and X. Li (2008), Radial gradients of phase space density of the outer radiation belt electrons prior to sudden solar wind pressure enhancements, Geophys. Res. Lett., 35, L18101, doi:10.1029/2008GL034866.

Turner, D. L., V. Angelopoulos, Y. Shprits, A. Kellerman, P. Cruce, and D. Larson (2012), Radial distributions of equatorial phase space density for outer radiation belt electrons, Geophys. Res. Lett., 39, L09101, doi:10.1029/2012GL051722. 